\title{
Current Probes, More Useful Than You Think
}

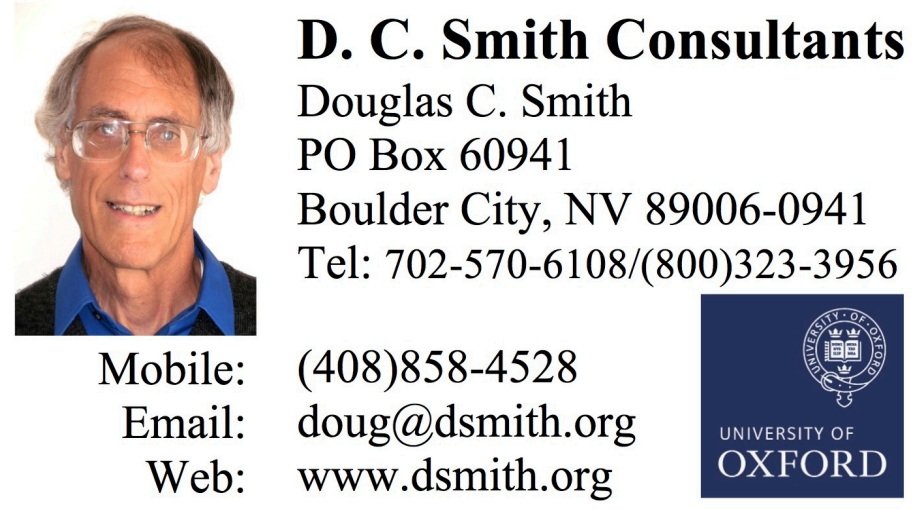

Training and design help in most areas of Electrical Engineering

Copyright (C) 1998 Institute of Electrical and Electronics Engineers. Reprinted from the IEEE 1998 EMC Symposium Proceedings.

This material is posted here with permission of the IEEE. Internal or personal use of this material is permitted. However, permission to reprint/republish this material for advertising or promotional purposes or for creating new collective works for resale or redistribution must be obtained from the IEEE by sending a blank email message to info.pub.permission@ieee.org.

By choosing to view this document, you agree to all provisions of the copyright laws protecting it. 


\title{
Current Probes, More Useful Than You Think
}

\author{
Douglas C. Smith \\ Auspex Systems \\ 2300 Central Expressway \\ Santa Clara, CA 95050 \\ Phone: (408) 566-2157
}

\begin{abstract}
Current probes have been used by EMC engineers for measuring common mode currents to track down EMC problems for many years. Based upon analysis of current probe operation, new uses and ways to extend probe performance are presented. Methods discussed include relative phase measurements, extended low frequency performance, and direct measurement of conductor voltage drop.
\end{abstract}

\section{Current Probe Operation}

\section{Equivalent Circuit}

Understanding current probe operation can be made easier by using equivalent circuits. Figure 1 below shows a simplified equivalent circuit of a current probe [1]. We will add to it later, but even this simple model is useful in analysis of current probes.

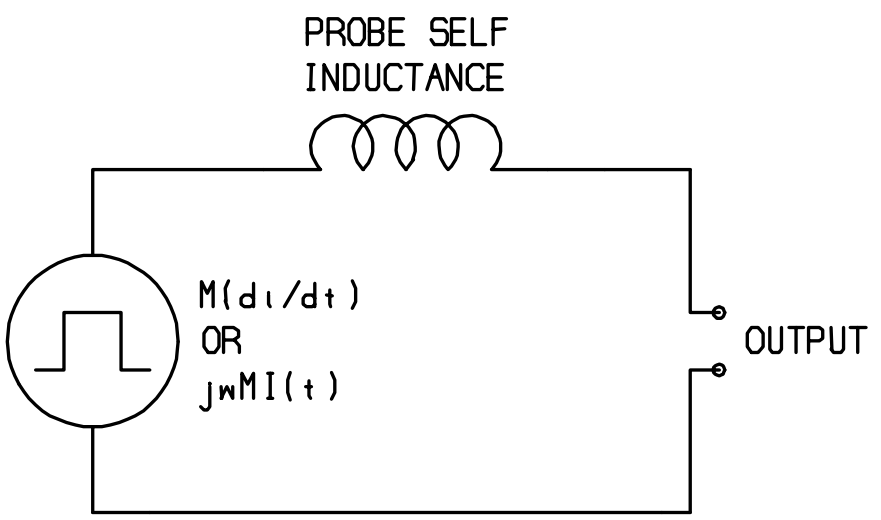

Figure 1. Current Probe Equivalent Circuit (simplified)

The simplified Thevenin equivalent circuit consists of an open circuit voltage and a series impedance. The open circuit voltage is equal to $\mathbf{M d i} / \mathbf{d t}$, where $\mathbf{M}$ is the mutual inductance between the probe pickup coil and the current carrying wire to be measured and $\mathbf{d i} / \mathbf{d t}$ is the time derivative of the current flowing in the wire. The Thevenin series impedance consists of the probe's pickup coil inductance. In the frequency domain, the Thevenin voltage, Mdi/dt, becomes $\mathbf{j} \omega \mathbf{M i}(\mathbf{t})$. The assumption is made that the current probe does not significantly affect the current flowing in the wire. Normally this is a reasonable assumption as long as the probe is terminated in $50 \Omega$ or less [2].
For a constant current swept in frequency, the open circuit voltage rises linearly with frequency at $20 \mathrm{~dB} /$ decade. The probe pickup coil inductance however forms a low pass $L / R$ filter with the external termination, usually $50 \Omega$. This filter has a flat response up to the corner frequency of $50 \Omega$ and the inductance of the probe pickup coil. After that the filter response falls off at $20 \mathrm{~dB} /$ decade. Both of these effects are shown in Figure 2 [1].

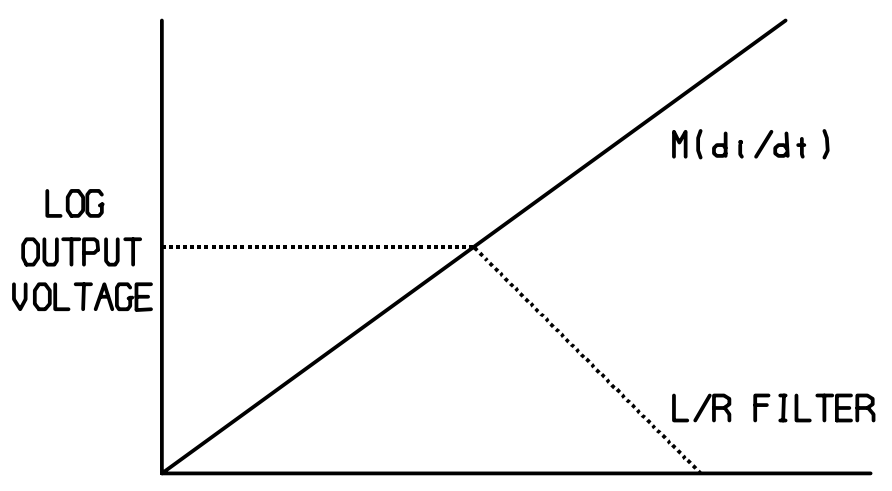

Figure 2. Thevenin Circuit Frequency Response

When the open circuit voltage and the filter response are combined, the result (for a constant measured current) has a sloping low frequency region and a flat high frequency region above the corner frequency as shown in Figure 3 [1].

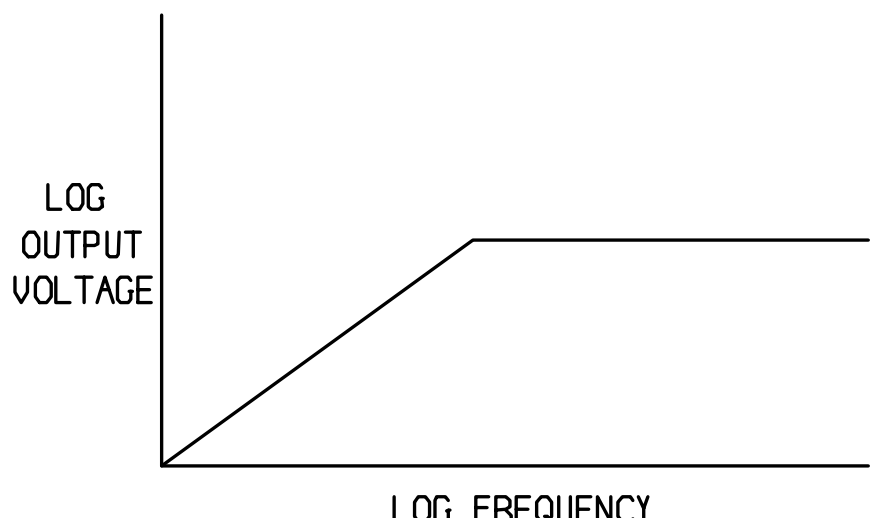

Figure 3. Current Probe Frequency Response 
The output voltage of a current probe is related to the current passing through it by its transfer impedance, $\mathbf{Z}_{\mathbf{t}}$. Thus a probe having a $1 \Omega$ transfer impedance will have a 1 volt output for a current passing through of 1 amp. Figure 3 is also the plot of transfer impedance of our example current probe.

\section{Core Losses}

The core losses of the current probe represent an additional impedance in parallel with the output of the probe [3]. For our purposes here, a pure resistance independent of frequency will be used. This assumption is close enough to reality to yield a greater understanding of current probe operation and to get some useful results.

Figure 4 shows the current probe equivalent circuit with an additional resistance across the output to represent core losses. The resulting frequency response is shown in Figure 5 [1].

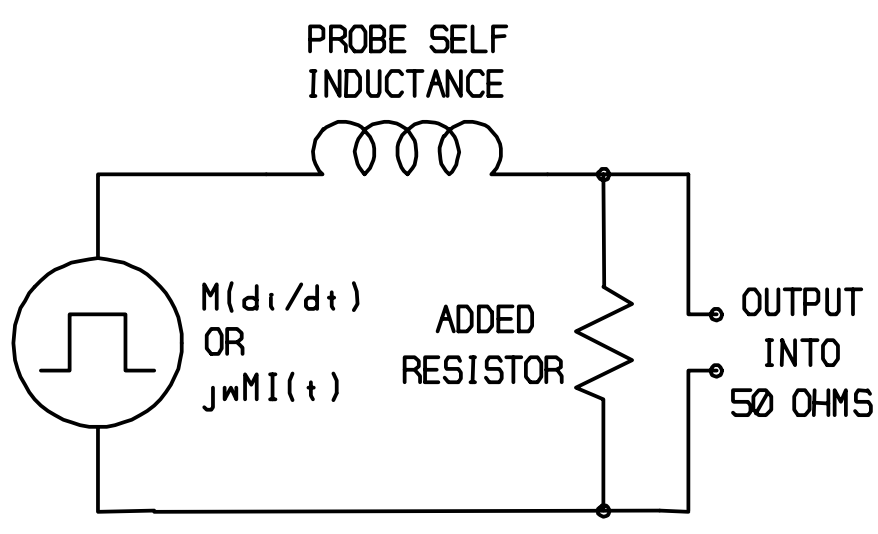

Figure 4. Current Probe Model With Core Losses

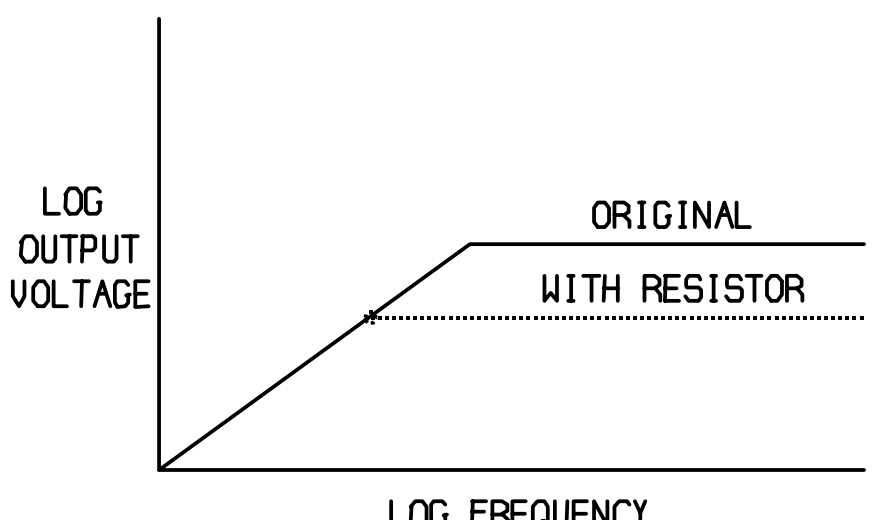

Figure 5. Probe Frequency Response With Core Losses

The added resistor does not affect the open circuit Thevenin voltage, but it does lower the corner frequency of the $L / R$ filter. The inductive reactance of the current probe self inductance equals the effective (lower) load resistance at a lower frequency. So it can be seen that the effect of core loss is to lower the high frequency sensitivity of the current probe.

\section{EXTENDing Low FreQuenCy RESPONSE}

The effect on probe frequency response of a parallel resistor on the output of the probe can be used to advantage. By adding parallel resistance at the output of the probe, the flat region of frequency response can be extended to lower frequencies at the expense of probe sensitivity.

From Figure 5 we would expect that the high frequency sensitivity of a current probe should be proportional to the load resistance. For example, if the load resistance is halved, so is the corner frequency. Since the slope of the open circuit voltage is $6 \mathrm{~dB} /$ octave, halving the corner frequency also halves the probe output voltage above the corner frequency.

This effect is shown in Figures 6 and 7 [1]. Figure 6 shows a square wave current of about $6 \mathrm{MHz}$ through a Fisher Custom

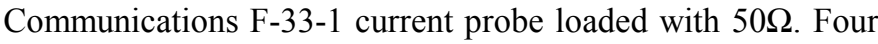
$50 \Omega$ feed through terminations were added at the probe output to form a $10 \Omega$ load on the probe for Figure 7.

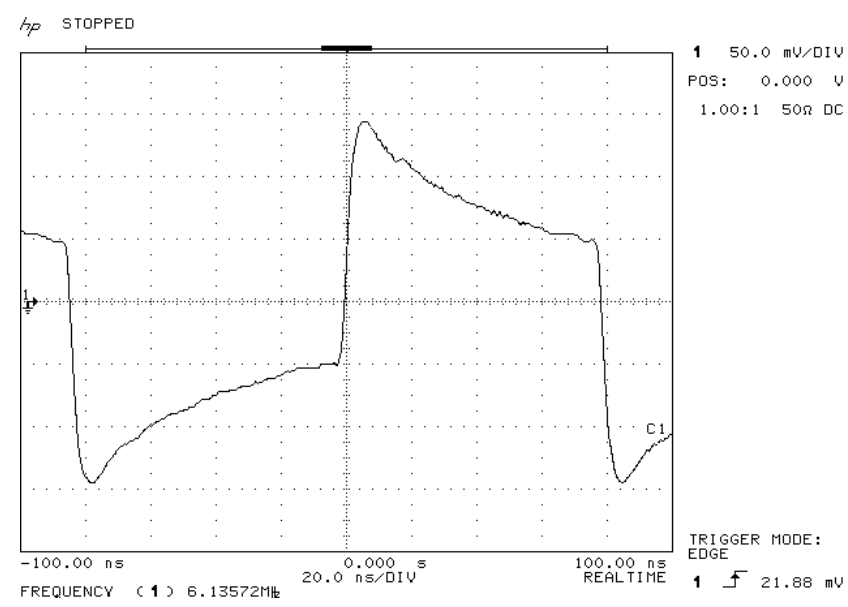

Figure 6. F-33-1 Output for Square Wave Current (39 $\mathrm{mA}$ at $\sim 6 \mathrm{MHz}$ )

The significant "droop" in the square wave of Figure 6 is due to the fact that $6 \mathrm{MHz}$ is near the corner frequency of the F-331 probe. Above that frequency, the probe has flat frequency response as in Figure 3.

The F-33-1 probe has a transfer impedance in the flat region of about $5 \Omega$. The edges of the square wave of Figure 6 have an amplitude of about $194 \mathrm{mV}$, which yields a current change of about $40 \mathrm{~mA}$. 
In Figure 7, one sees that the waveform is indeed smaller with less droop, just as expected for a $10 \Omega$ load on the probe.

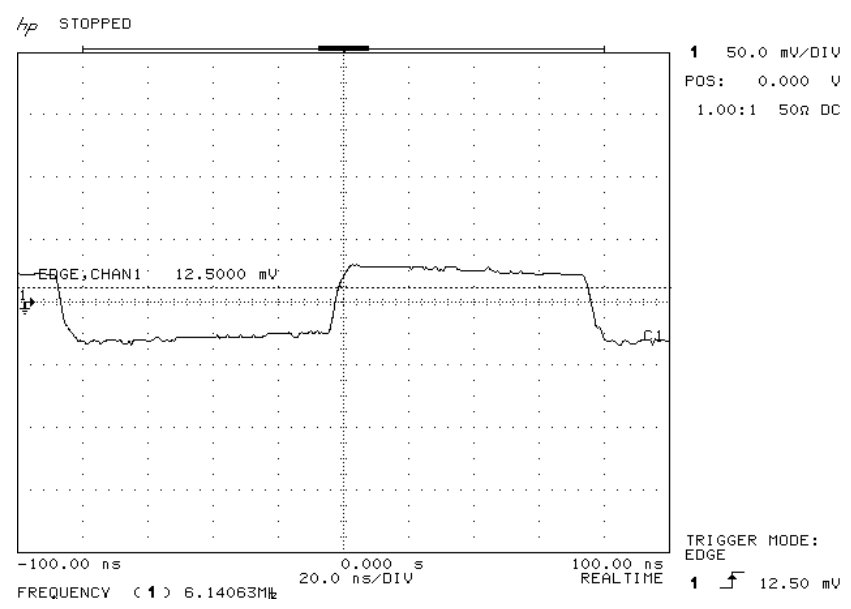

Figure 7. F-33-1 Probe Output With $10 \Omega$ Load (40 $\mathrm{mA}$ at $\sim 6 \mathrm{MHz}$ )

Figure 8 expands the scale of Figure 7 to $10 \mathrm{mV} /$ division so its amplitude can be measured [1]. It can be seen that the amplitude of the edges is about $50 \mathrm{mV}$. This gives a transfer impedance of $1.25 \Omega$. The amplitude and transfer impedance ratios for the $50 \Omega$ and $10 \Omega$ cases are equal to $4(5 / 1.25)$.

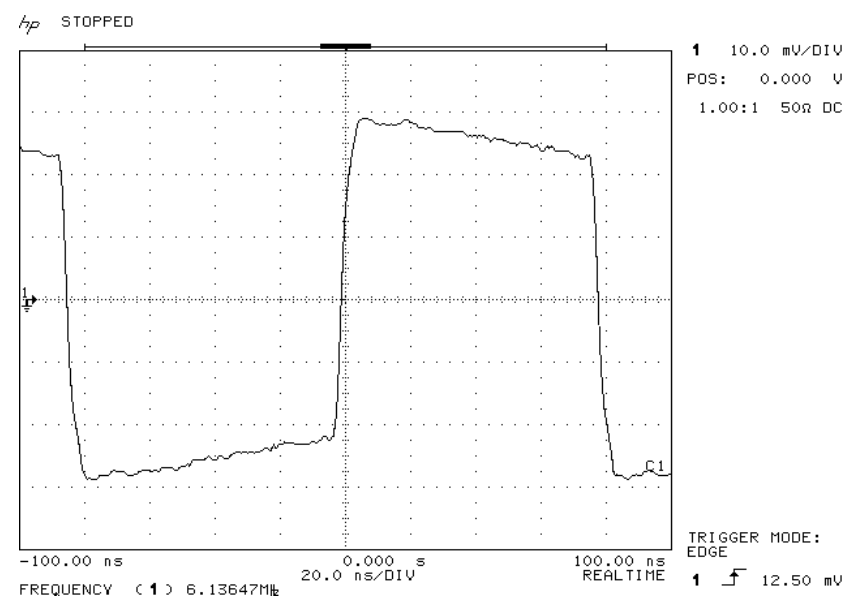

Figure 8. F-33-1 Probe Output With 10 $\Omega$ Load (40 $\mathrm{mA}$ at $\sim 6 \mathrm{MHz}$ )

But, $50 \Omega$ and $10 \Omega$ were the actual load impedances, a ratio of 5 not 4 ! The difference is explained by the core losses of the probe. The core loss is in parallel with the external load so the equation 1 must be true:

$$
\begin{aligned}
& 50 \| \mathrm{R}_{\mathrm{c}}=4\left(10 \| \mathrm{R}_{\mathrm{c}}\right) \\
& \text { where: } \\
& \quad \bullet \| \text { means "in parallel with" } \\
& -\mathrm{R}_{\mathrm{c}} \text { is the core loss resistance }
\end{aligned}
$$

Equation (1) is satisfied with a core loss of about $150 \Omega$.

\section{Point to Remember}

Loading a current probe output with a lower resistance does indeed extend its low frequency response at the expense of high frequency sensitivity. This technique can be used to either modify a current probe's frequency response or to approximately measure the core losses of the probe.

\section{Conductor Voltage Drop Measurement}

Current probes can be used to directly measure, with flat frequency response, the inductive voltage drop across conductors. Referring to Figure 3, current measurements are easily made in the flat region of the probe's current response. Time domain current measurements require a flat frequency response in order to accurately reproduce the time domain waveform. However, below the corner frequency, in the sloped region of the current frequency response, voltage measurements can be made.

In the sloped region of frequency response, the output of the probe is equal to Mdi/dt. The inductive drop across the conductor carrying current through the probe is $\mathbf{L d i} / \mathbf{d t}$, where $\mathbf{L}$ is the inductance of the wire per unit length. If one can calculate or measure the mutual inductance between the current probe and the conductor, $\mathbf{M}$, and the inductance of the wire, $\mathbf{L}$, one can calculate the voltage drop across the conductor per unit length.

For example, suppose $\mathbf{M}$ is $80 \mathrm{nH}$ and $\mathbf{L}$ is $10 \mathrm{nH} / \mathrm{cm}$. Then the output of the probe in the sloped region of its frequency response is 8 times the inductive drop across each centimeter of conductor!

Is there an easy way to determine M? Yes, there is. In the flat frequency response region, a current probe's transfer impedance is given by equation 2 [2]

$$
\begin{gathered}
\mathrm{Z}_{\mathrm{T}}=\left(\mathrm{M} / \mathrm{L}_{\mathrm{P}}\right) \mathrm{R}_{\mathrm{L}} \\
\text { where: }
\end{gathered}
$$

- $\mathbf{Z}_{\mathbf{T}}$ is the probe transfer impedance

- $\mathbf{R}_{\mathbf{L}}$ is the load resistance at the probe output, usually $50 \Omega$.

- $\mathbf{L}_{\mathbf{P}}$ is the self inductance of the current probe's pickup coil. 
The current probe self inductance, $\mathbf{L}_{\mathbf{P}}$, can be easily determined. At the corner frequency of the current probe's response, the probe's self inductive reactance is equal to the load resistance, $\mathbf{R}_{\mathbf{L}}$. Since the corner frequency, $\mathbf{F}_{\mathbf{C}}$, and load resistance are known, $\mathbf{L}_{\mathbf{P}}$ is given by equation 3 .

$$
\mathrm{L}_{\mathrm{P}}=\mathrm{R}_{\mathrm{L}} /\left(2 \pi \mathrm{F}_{\mathrm{C}}\right)
$$

Equation (2) can now be solved for $\mathbf{M}$.

An example of using a current probe to measure conductor voltage would be to measure the voltage drop across an equipment grounding conductor during a simulated lightning surge. If the mutual inductance of the current probe, $\mathbf{M}$, is 100 $\mathrm{nH}$ and the inductance of the wire is $10 \mathrm{nH} / \mathrm{cm}$, the probe output would be the voltage drop across each $10 \mathrm{~cm}$ of cable. The voltage drop across the length of the cable is then the output of the probe multiplied by 10 times the length of the cable in meters. An assumption is made here that the cable length is electrically shorter than the $10 \mu \mathrm{sec}$ risetime of the surge.

For the case of lightning surge, the F-33-1 current probe's sloped region of response, which extends up to about $3 \mathrm{MHz}$, is well past the bandwidth of a surge with a $10 \mu \mathrm{sec}$ risetime. The F-33-1 current probe is well suited for this measurement up to its saturation current of about 100 amps.

Being able to make a voltage measurement across a conductor, as in this example, can be very useful. This is especially true if the conductor is carrying high voltage.

\section{Point to Remember}

Current probes can be used to directly make inductive drop voltage measurements if the bandwidth of the current in the conductor is below the corner frequency of the probe's frequency response.

\section{Differential Current Measurements}

Current probes can also be used to measure the relative phase and amplitude between two common mode currents. Such a "differential" current measurement can provide information on how to fix an EMC problem in a piece of equipment. This technique will require a set of current probes with responses matched to within a few percent, a signal combiner, and a spectrum analyzer.

\section{Matched Current Probes}

Most current probes are specified to have an amplitude accuracy of $\pm 2 \mathrm{~dB}$. This is adequate for most measurements, but not for cases where the output of one probe is to be subtracted from the output of another as in the method to be described below. If one probe has a transfer impedance that is $20 \%$ higher than another one, the result of subtracting their outputs will not be very useful. If two such probes were placed on the same conductor, the subtraction of their outputs would yield a "common mode" rejection of only a few $\mathrm{dB}$.

The amplitude responses of the F-33-1 probes I use for comparative measurements are matched to within $1 \%$ to $2 \%$.

\section{Using a Signal Combiner With Matched Current Probes}

If two current probes are connected to a signal combiner, the output of the combiner will be the sum or the difference of the currents flowing through the current probes depending of the orientation of the current probes and the type of combiner. I often use a $180^{\circ}$, subtracting, combiner made by Mini-Circuits (model ZFSCJ-2-1). A $0^{\mathrm{O}}$, adding, combiner will work equally well if one of the current probes is reversed on its measured conductor.

When two current probes are connected to a signal combiner, it is important to use identical cables and to add $50 \Omega$ feedthrough terminations at the output of each current probe. Identical cables preserve the relative phase between the signals from the current probes whereas the terminations insure the combiner sees $50 \Omega$ on all of its ports.

The input impedance of a current probe is, to a first order approximation, the inductive reactance of its internal coil. In the flat region of the probe's frequency response, this inductive reactance exceeds $50 \Omega$. So by adding a $50 \Omega$ feedthrough termination to the probe output, its output impedance becomes $50 \Omega$ in this frequency region.

It is necessary to take into account the loss of the combiner and the $6 \mathrm{~dB}$ reduction in the output of each current probe resulting from the $50 \Omega$ termination added to its output (total load on the probe is $25 \Omega$ ). For a pair of F-33-1 probes, a $50 \Omega$ termination on each, connected to a Mini-Circuits ZFSCJ-2-1 $180^{\circ}$ combiner, the transfer impedance of each probe will be about $1.5 \Omega$.

\section{Differential Current Measurement}

Using a pair of matched current probes and a signal combiner connected to a spectrum analyzer, the relative phase and amplitude of two currents are easily measured. The apparatus should first be checked by doing a "null experiment." This can be done simply by just clamping one current probe on a wire carrying RF currents. When the second probe is clamped on the same wire adjacent to the first probe and in the same orientation, the combiner output will reduce by $20 \mathrm{~dB}$ to $30 \mathrm{~dB}$ over a broad frequency range when a $180^{\circ}$ combiner is used.

After the apparatus is checked, one is ready for serious EMC troubleshooting. An example of what is possible is shown in Figure 9 [1]. In this example, the EUT is relatively small with respect to a wavelength at the frequencies of interest so that most of the radiation is from the cables. 
The EUT of Figure 9 has three cables, two signal cables and one power cable. Upon installing a current probe on signal cable 1, the spectrum of Figure 10 is observed [1]. If the common mode current at frequency $\mathbf{f 5}$ is too high, what can be done to reduce it?

It would be very useful to know if signal cable 2 is carrying the same current or if the other half of the circuit involves the power cord. This knowledge will help the troubleshooting process.

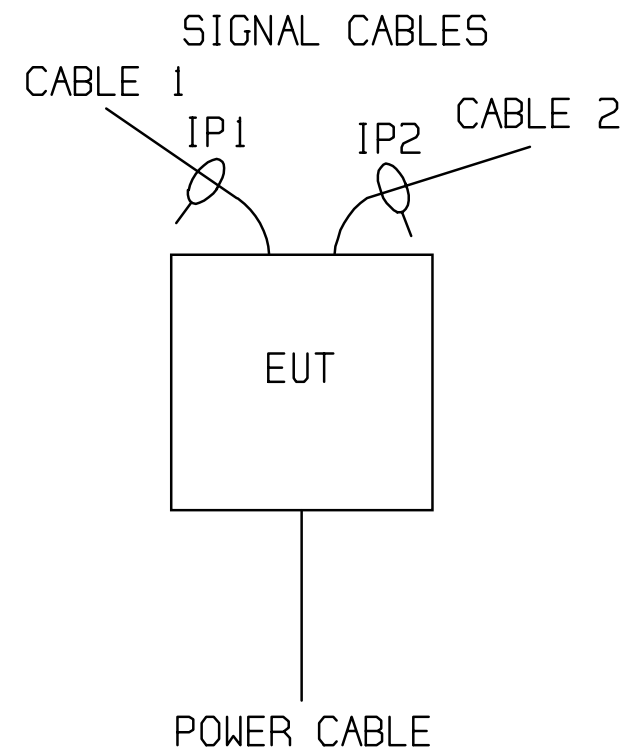

Figure 9. Hypothetical EUT With Common Mode Cable Currents

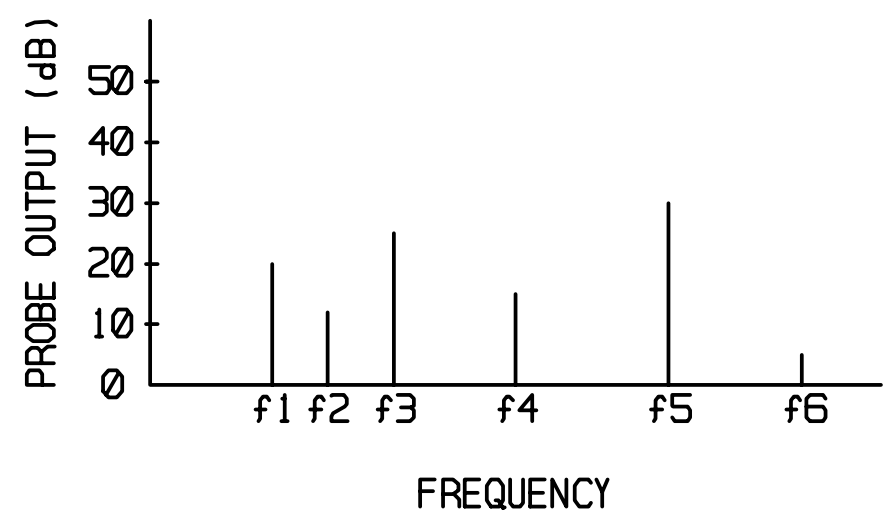

Figure 10. Signal Spectrum of CM Current on Signal Cable 1

The second current probe is then installed on signal cable 2 in the same orientation. In order to make it easier to determine current probe orientation, I suggest putting a red dot on the same face of both probes. In this example, the red dots would both be facing to the right or both to the left. Since the probes are matched and I use a $180^{\circ}$ combiner, if the same current is flowing from left to right from signal cable 1 to signal cable 2 , that current will produce the same output in both probes.
The result will cancel in the combiner, reducing its output. For our example, when the second probe is installed, the spectrum of Figure 11 is produced [1].

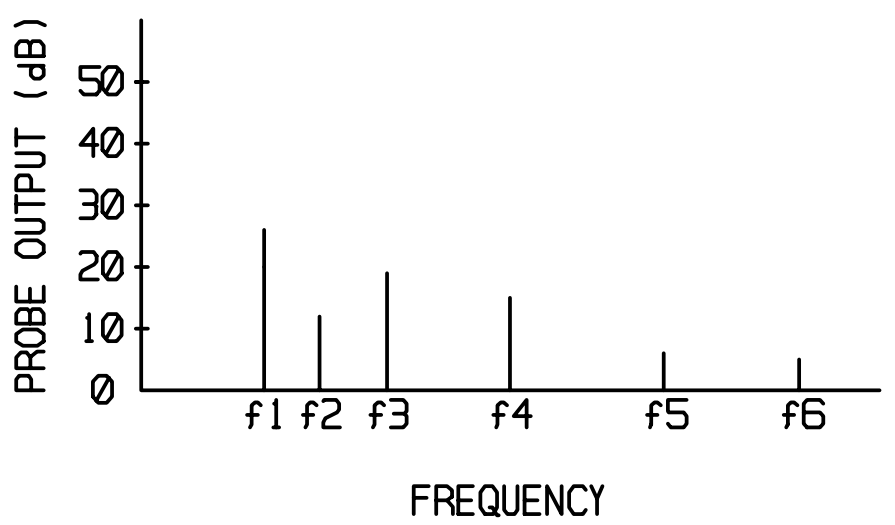

Figure 11. CM Current on Signal Cable 1 Minus CM Current on Signal Cable 2

\section{Interpreting Measurement Results}

The net current at frequency f1 increased by $6 \mathrm{~dB}$. This indicates that the outputs of the two current probes are similar in amplitude but of opposite phase (remember a $180^{\circ}$ combiner is being used). Accordingly, the currents in the wires are flowing in opposite directions with respect to the probes. Since the "red dots" of the current probes are facing in the same direction, both left or both right, the currents are flowing either both into the EUT or from it. At frequency f1, the two common mode signal currents must be added together and exiting on the power cable, the only other cable on the EUT.

At frequency f3, the net output of the combiner is reduced somewhat. This indicates the two currents have a component flowing in the same direction (right to left or vice versa)), but not all of the current from cable 1 flows to cable 2 .

At frequency f5, there is a strong null of almost $30 \mathrm{~dB}$ when the second current probe is added. This indicates that the currents at this frequency on cables 1 and 2 are nearly identical in amplitude and direction (say from right to left). This is because the current probe outputs are of nearly the same phase and amplitude and are being subtracted in the combiner.

The net current at other frequencies (f2, f4, and f6) remains unchanged indicating that the current on cable 1 does not significantly appear on cable 2 . These currents must either radiate from the EUT (which we have assumed is not happening for this example) or must appear on the power cable.

The current flowing on the signal cables at frequency f5 is passing from one cable through the EUT and onto the other 
cable in the same direction relative to the drawing. This indicates there is a driving source between the ground references of the two signal cables driving them like a dipole. The fix would be to "stiffen" up the ground between the two signal ports to reduce the driving source voltage.

This fix would probably have no effect at frequency f1 because at that frequency, current is flowing in on the signal leads and exiting on the power cable. The problem at f1 is a driving voltage between the signal leads and the power cable.

This information can be used to track down the mechanisms in the EUT that are generating the common mode currents.

\section{Null EXPERIMENTS}

It is important to check current probes for proper operation. This especially true when measuring currents due to ESD or other transient events. I do this with null experiments. Two possible methods are shown in Figures 12 and 13 [1].

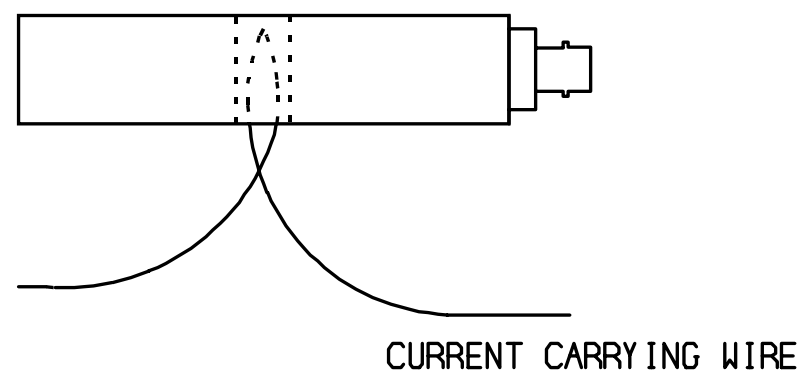

Figure 12. Folded Wire Null Experiment

Figure 12 shows a "folded wire" null experiment where the current carrying wire is folded and inserted into the probe. The current probe is thus exposed to the electric field on the wire but with no net current. Its output should be zero or null! To the extent the probe output is not zero, the output is the error in the current measurement. Possible sources include inadequate electric field shielding in the current probe and leakage through the shield transfer impedance of the probe cable.

Figure 13 shows a dual reversed probe null experiment. The outputs of the two probes should have nearly equal amplitude but opposite phase. If the two outputs are added in a combiner or an oscilloscope, the result should be nearly zero and represents the error in the measurement.

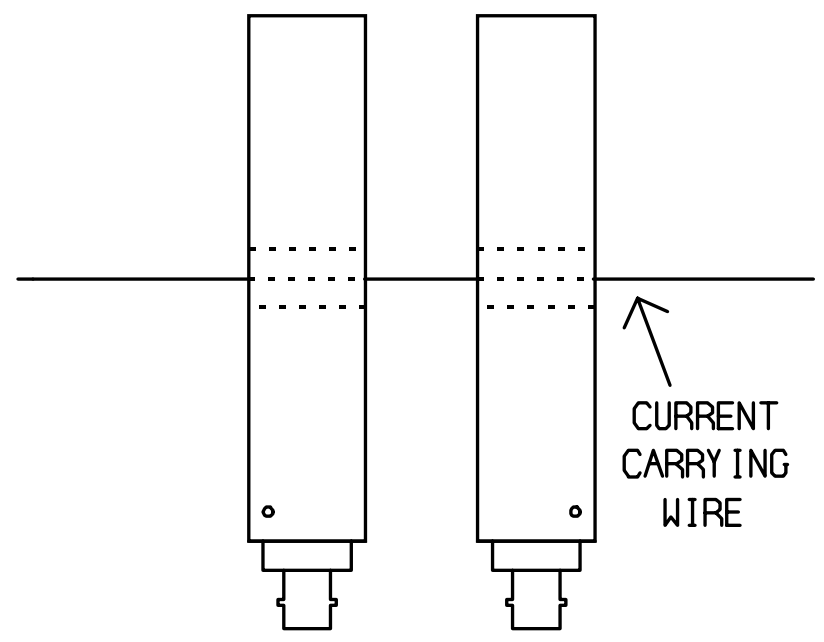

Figure 13. Dual Reversed Probe Null Experiment

Take care to keep the current probes as close together as possible to minimize phase shift in the current between the probes. Similarly, in the folded wire null experiment, the wire should not protrude past the current probe body or phase shift along the wire will result in an undesired output.

\section{Conclusions}

By understanding how current probes work, they can be used for new purposes or their performance extended. This paper has shown how to extend low frequency response of current probes, use them for induced voltage measurements, and for measuring relative phase and amplitude between two currents (differential current measurements).

Current probes remain a very useful tool available to EMC personnel for finding and correcting EMC problems in equipment designs. Hopefully, this paper has extended the usefulness of current probes even further.

\section{REFERENCES}

[1] Figure from the manuscript of Troubleshooting Electronic Circuits and Designs Using High Frequency Measurements, Douglas C. Smith, to be published in late 1998. Used with permission.

[2] High Frequency Measurements and Noise in Electronic Circuits, Douglas C. Smith, VanNostrand Reinhold, 1993, pp. 165-167.

[3] Private conversation with Joe Fischer of Fischer Custom Communications. 